\title{
Telluric sodium layer temporal variations
}

Mark Chun, Tim Butterley, Richard Wilson, Remy Avila, Jose-Luis Aviles, et al.

Mark Chun, Tim Butterley, Richard Wilson, Remy Avila, Jose-Luis Aviles, Brent Ellerbroek, Francois Rigaut, "Telluric sodium layer temporal variations," Proc. SPIE 7015, Adaptive Optics Systems, 70154V (14 July 2008); doi: 10.1117/12.787633

Event: SPIE Astronomical Telescopes + Instrumentation, 2008, Marseille, France 


\title{
Telluric sodium layer temporal variations
}

\author{
Mark Chun $^{* a}$, Tim Butterley ${ }^{\mathrm{b}}$, Richard Wilson ${ }^{\mathrm{b}}$, Remy Avila ${ }^{\mathrm{c}}$, \\ Jose-Luis Aviles ${ }^{\mathrm{c}}$, Brent Ellerbroek ${ }^{\mathrm{d}}$, Francois Rigaut ${ }^{\mathrm{e}}$ \\ ${ }^{a}$ Institute for Astronomy, University of Hawaii, 640 N. A'ohoku Place, Hilo, HI 96720; \\ bUniversity of Durham; 'Universidad Nacional Autónoma de México; \\ dThirty Meter Telescope Project; ${ }^{\mathrm{e}}$ Gemini Observatory
}

\begin{abstract}
We present observations of the high-speed variations of the altitude of the telluric sodium layer. In this experiment we observed the Gemini-North sodium laser guide star from approximately 80 meters off-axis using the UH-2.2m telescope on Mauna Kea, Hawaii. Observations were made using an electron-multiplying camera at a rate of about $100 \mathrm{~Hz}$. The temporal power spectrum of the layer centroid follows a power law between 0.001 and $1 \mathrm{~Hz}$ and we find that the exponent of the power law $(\alpha=-1.8)$ is similar to that found at lower temporal frequencies from lidar experiments. This data set taken with the lidar results shows that the power spectrum of the sodium layer mean altitude follows a simple power law over 5 orders of magnitude from $10^{-4.5} \mathrm{~Hz}$ to $1 \mathrm{~Hz}$. The approach taken in this experiment is difficult due to telescope jitter in any of the three telescopes (Gemini-N, Gemini-N LGS launch telescope, or from the observing $\mathrm{UH} 2.2 \mathrm{~m}$ ) and atmospheric tip/tilt wave front aberrations. We circumvented these problems by analyzing the differential motion between two distinct features that appeared in the sodium layer during that night.
\end{abstract}

Keywords: telluric sodium, sodium laser guide stars

\section{INTRODUCTION}

Sodium laser guide stars have become common components of adaptive optics systems on large ground-based astronomical telescopes. Their use in extending the sky coverage of high-order adaptive optics systems is key to implementing adaptive optics systems on the next generation of large groundbased telescopes. One challenging aspect of these new systems arises from the fact that there is an ambiguity between the lowest order wave front modes introduced by the atmosphere and variations in the sodium layer altitude and profile. These ambiguities are resolved in current laser guide star adaptive optics systems by using an auxiliary natural guide star wave front sensors to monitor the true wave front aberrations for these lowest modes. These wavefront sensors typically operate at a reduced temporal bandwidth in order to preserve the large sky coverage afforded by the laser guide star. As such, these wavefront sensors do not monitor rapid variations in the sodium layer. As the telescope diameters increases the residual wavefront error due to these differences increases dramatically and any error between the truth wavefront sensor and the higher-order LGS wavefront sensor could dominate the residual wavefront error budget ${ }^{1}$. A key missing input to the design of these systems is the amount of variation in the sodium layer at frequencies between the NGS and LGS wavefront sensors (e.g. $10^{-2}$ to $10^{3} \mathrm{~Hz}$ ).

To date the main source of information on the telluric sodium layer variations in this context comes from lidar experiments ${ }^{2}$. These observations show that the power spectral density of the mean altitude of the sodium layer follows a power law with an exponent of -1.8 between $10^{-4}$ and $10^{-2.5} \mathrm{~Hz}^{2}$. This

\footnotetext{
*mchun@ifa.hawaii.edu; phone 1808 932-2318; fax 1808 932-2317
}

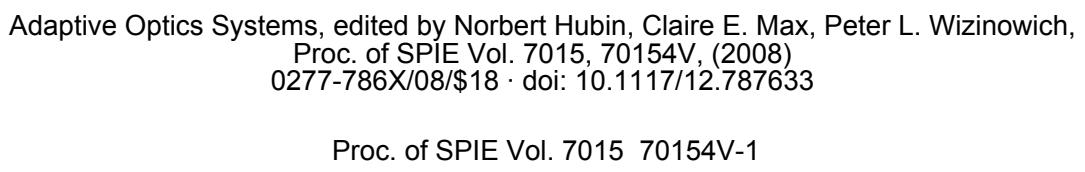


result represents the best information to date on the temporal variations of the sodium layer but samples a frequency region that is still at least an order of magnitude lower than needed.

\section{OBSERVATIONS AND DATA}

\subsection{Experimental setup}

To address the question of the the higher frequency variability of the sodium laser guide star we observed the Gemini-North sodium laser guide star (LGS) on Mauna Kea, Hawaii from off-axis and monitored the sodium layer profile variations. On the night of 24 September 2007 (UT) we observed the Gemini-North LGS using the University of Hawaii 2.2-meter telescope (UH2.2m) located 81.8 meters south of the Gemini-North telescope. During this night the Gemini telescope was performing science queue observations with their LGS adaptive optics system while the UH telescope was equipped with a SLODAR and Generalized SCIDAR (GS) experiment (see Butterley et $\mathrm{al}^{3}$ in these proceedings). The SLODAR/GS experiment used an electron-multiplying CCD camera mounted on a rotating stage at the Cassegrain focus of the telescope. For the LGS observations we removed the SLODAR's lenslet array but retained its plate scale reducer. This provided a plate scale of $0.295 \% /$ pixel on the $2 \times 2$ binned pixels of the EMCCD detector and a field of view of $151 \times 151$ arcseconds to image the LGS. To increase the frame rate of the images to $100 \mathrm{~Hz}$, we aligned the elongated laser guide star beam with the rows of the camera detector and only read out a region 40 and 300 pixels (binned 2x2). Individual images had an exposure time of 4 milliseconds which, in the reduced LGS profiles, gave about 400 e- per binned pixel at the peak of the profile.

\subsection{Data and data reductions}

The data were collected in packets of 5000 consecutive frames so data sets were saved at a rate of about once per minute. Several data packets were collected in sequence until the beam drifted away from the center of the detector or the rotation of the beam in the detector changed. The data presented encompass one and a half hours between 12.5-14 hours (UT). The data set does not have complete coverage of this period and includes several gaps of 10-15 minutes when the Gemini queue observations offset from their science program target position for sky observations. The data is evenly sampled within the 5000 samples in the data packet and unevenly sampled between these data sets.

The basic packet of data consisted of a sequence of images of the laser guide star aligned along rows of the detector and sky on either side of the laser guide. The centroid and full-width half-maximum (FWHM) of the LGS profile perpendicular to the propagation direction was calculated for each image. In this direction, the average FWHM is 3."1 or 10.6 pixels. A DC sky value was calculated for each image in the data set by calculating a median value in the pixels at the edges of the field of view. Each two dimensional image was then compressed into a single dimension by summing the rows within one FWHM of the center row (typically $\sim 20$ rows were summed). This number of rows to sum maximized the signal-to-noise ratio of the profiles. Each data packet was therefore reduced to 5000 one dimensional profiles of the laser guide star along the propagation direction. Over the course of the observations the elevation angle of the telescope changed from 75 to 55 degrees. This amounts to a small correction to the projection of the LGS onto to altitude and is made for each individual data set based on the observation elevation angle. No correction for rotational misalignment of the LGS along rows were made but the rotation stage was adjusted manual whenever telescope pointing adjustments were made.

The detector was an EMCCD with an electron multiplying gain of 120 so while the read noise is negligible we have an increase in photon noise of about a factor of two due to noise in the gain 
multiplying stages. Taking this and the intrinsic gain of the readout electronics into account (3.02 e-/ADU) the signal-to-noise ratio of individual profiles averages slightly better than 10 at the peak of the profile. The average total signal-to-noise ratio for the beam profile is about 100. From this we estimate that our LGS beam centroid is no better than about $\sigma_{\mathrm{H}} \sim \mathrm{FWHM} / \mathrm{SNR} \sim 100 \mathrm{~m}$ for a nominal width of $10 \mathrm{~km}$.

The measurement error sets a limit on our accuracy of the sodium layer position but our ability to resolve structure within the sodium layer is limited by the atmospheric seeing. During this night we ran a SLODAR optical turbulence profiler on the Coude roof of the UH2.2m telescope. These data have an average seeing value of 0.72 " during the LGS observations. In this regime of $\mathrm{D} / \mathrm{r}_{0}(\sim 15)$ one would not expect a drastically improved resolution even at the short exposure times used for the LGS observations so we take the full seeing as a measure of our altitude resolution. This corresponds to an altitude resolution of $\Delta H \sim \frac{\epsilon_{F W H M} H^{2}}{\cos \left(\theta_{\text {zenith }}\right) d} \sim 360$ meters.

\section{ANALYSIS}

\subsection{Basic properties of the sodium layer}

Note that the mean altitude to the sodium layer is not known in this experiment as the elevation of the Gemini and UH2.2m telescopes were not recorded simultaneous with the data. For this reason have made the assumption that the mean altitude of the the sodium of layer is $90 \mathrm{~km}$ above sea level.

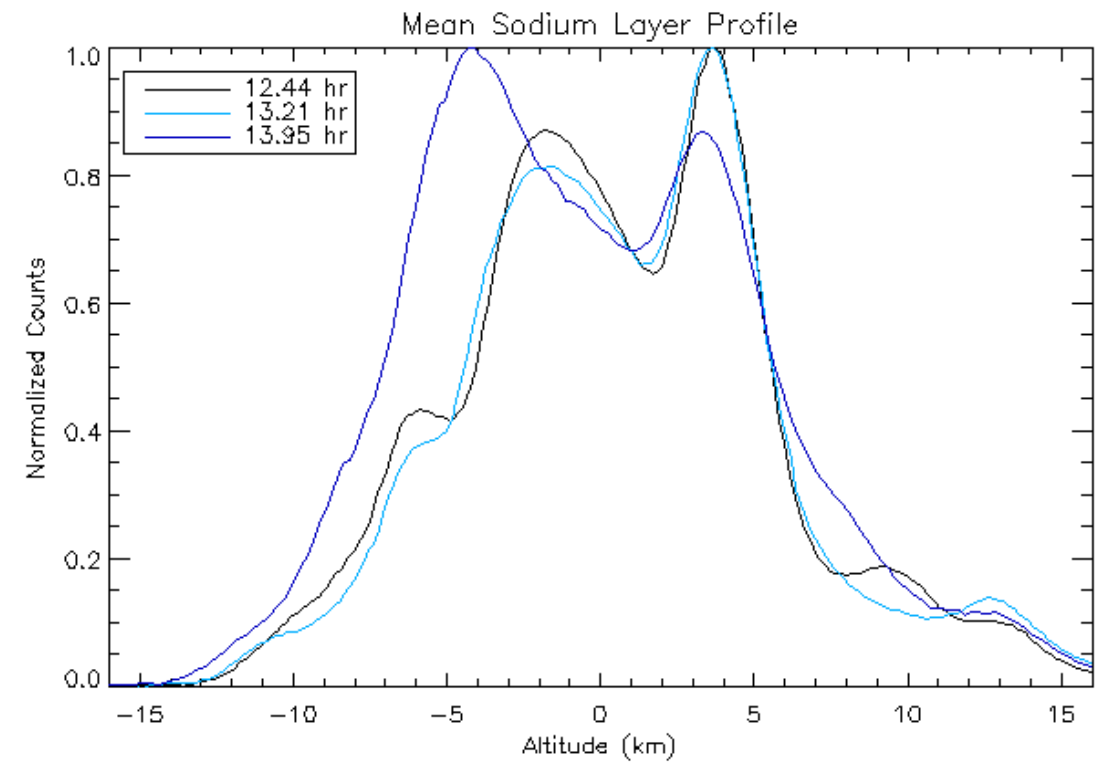

Illustration 1: Mean LGS profiles for the Mauna Kea, Hawaii on UT 24 September 2007. The three data sets span the observations and are each separated by approximately 45 minutes. The mean FWHM of the profile corresponds to $10 \mathrm{~km}$ assuming a mean altitude of $90 \mathrm{~km}$ above sea level. The peak of the profile corresponds to an average photon flux of $400 \mathrm{e}$-/pixel.

From the elongation of the laser guide star profile we estimate the mean layer thickness (FWHM) to be 10 kilometers with a one sigma variation of 580 meters over the 1.5 hour observation. The basic morphology of the profile shows multiple layers each with a FWHM of a few kilometer. On this particular night the profile was dominated by two distinct peaks within the layer. Figure 3 shows the mean profiles in three data packets spread out by approximately 45 minutes and spanning the observations. 


\subsection{Power spectral density of the mean altitude}

The quantity of interest for adaptive optics systems using sodium laser guide stars is the highfrequency changes in the mean altitude of the sodium layer. Herriot et al. (2006) ${ }^{1}$ have shown that high frequency variations in the range to the sodium layer can be misinterpreted as focus errors when using a laser guide star based wave front sensors. LIDAR experiments (Davis et $\mathrm{al}^{2}$ ) find that the power spectral density of the temporal variations of the mean altitude of the sodium layer is well described by a power law with an exponent $\alpha=-1.8$ between $10^{-4}$ and $10^{-2.5} \mathrm{~Hz}$.

Our data set provides insight into the higher temporal variations by sampling the sodium laser guide star profile at $100 \mathrm{~Hz}$. The centroids of the laser guide star profiles were calculated for the individual data sets provided continuous data for 5000 samples and data were collected over a period of 1.5 hours. In order to sample the lower frequencies we increased the length of the sampling by combining all data sets between telescope pointing offsets. For each laser guide star profile, a simple centroid was calculated to obtain the altitude of the sodium layer. An example of one of these data sequences is shown below in Figure 2.

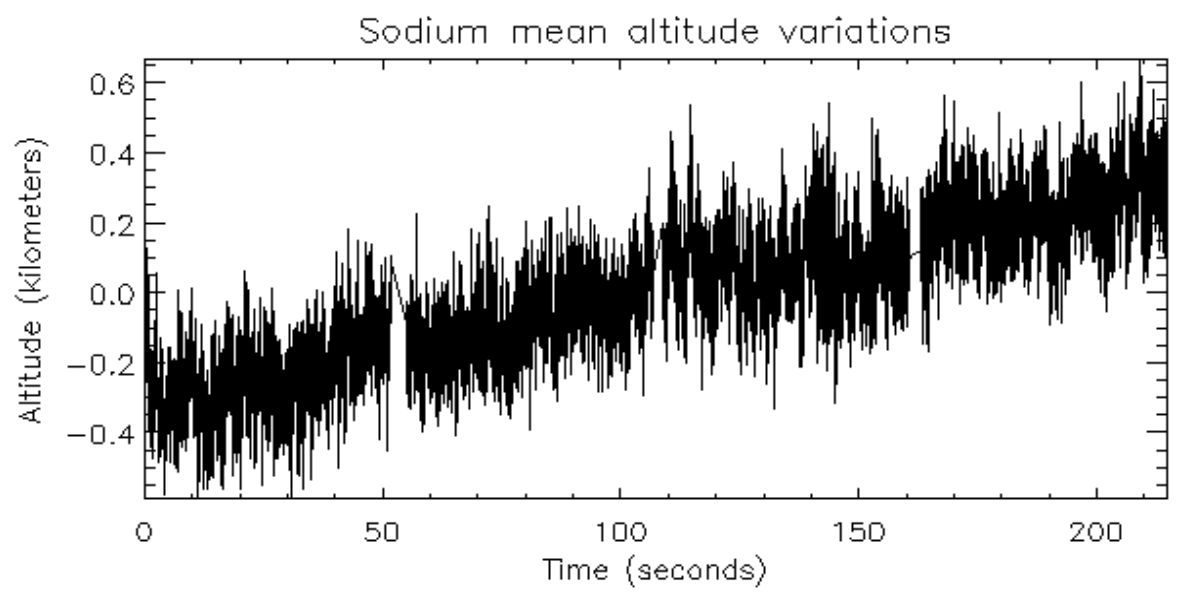

Illustration 2: An example of four consecutive data packets showing the variation of the mean altitude.

Figure 3 below shows the average power spectrum of the sodium mean altitude for this experiment. The data shown includes all data sets analyzed. Combining the data packets leads to an uneven temporal sampling of data and makes the normal method of calculating the power spectral density using FFTs problematic. To handle this we calculated the power spectrum as a Lomb periodogram ${ }^{4}$. This approach provides the power spectrum for an unevenly sampled data and has the addition benefit that aliasing of variations of temporal frequencies higher than the Nyquist frequency is minimized ${ }^{4}$. For the low frequency regime of our data we find a power law with an average exponent of $\alpha=-1.78$ with a standard deviations of 0.24 fits the data between $10^{-3}$ and $10^{-1} \mathrm{~Hz}$. These data overlap the data published by the lidar experiment and the low-frequency power law exponent is the same as found in that experiment. There is however a offset in the total variance by about an order of magnitude between the experiments. This could be an error in the scaling of the data or represent real variability in the total variance in the layer altitude at different sites or times.

At higher frequencies the variations of the mean height is complicated by atmospheric tip/tilt variations as well as telescope drift and jitter. Changes in the position of the beam along rows of the camera detector could be due to changes in the altitude of the telluric sodium or by motion in any of the telescopes (Gemini, Gemini LGS launch telescope, or the UH2.2m telescope). Telescope drift of 
the UH2.2m telescope is estimated from the SLODAR observations to be less than 1 arcsecond in 5 minutes unguided while the Gemini is likely to be better than this given its active pointing system. This is a significant amount of drift over the data sequences analyzed and could account for the slight turned down edge of the power spectrum in Figure 3. Telescope shake however is more insidious and and can manifest itself at discrete frequencies or as broader bumps in the average power spectrum. Indeed discrete frequencies can be seen at 10 and $15 \mathrm{~Hz}$ in the power spectrum of the sodium layer altitude and we believe these arise due to vibrations in one of the telescopes. These vibrations are seen in the power spectra of motion along the LGS beam as well as for motion perpendicular to the beam so the large spikes at these frequencies clearly do not arise from the sodium layer. In addition, there appears to be an excess variance between 0.1 and $1 \mathrm{~Hz}$ compared to the power law projected from lower frequencies. A power spectrum of the image motion generated from the SLODAR experiment on the UH2.2m on the same from night shows excess jitter power over a power law PSD between 3 and $8 \mathrm{~Hz}$.

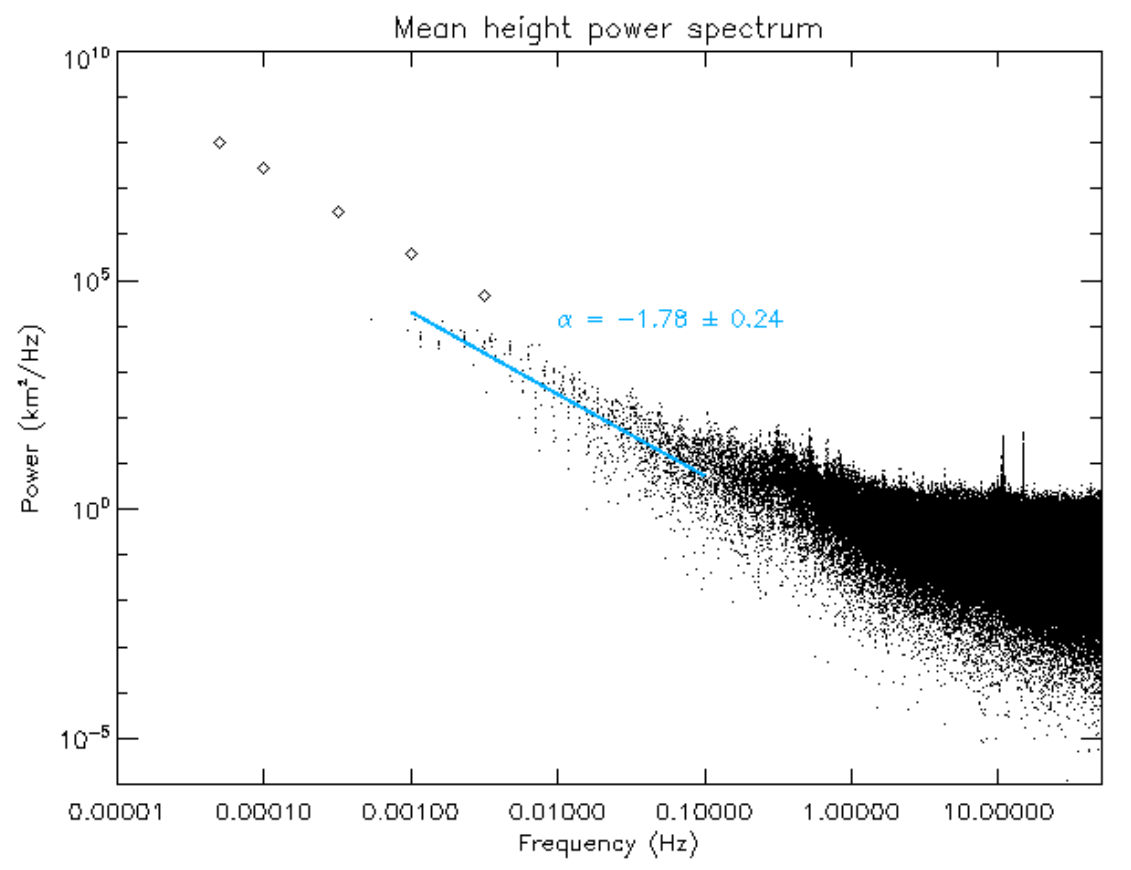

Illustration 3: Power spectrum of the mean altitude of the sodium layer above Mauna Kea on 24 September 2007 (UT). The diamonds represent the power law fit found by Davis et al. ${ }^{2}$ from the Colorado State University lidar data (not at Mauna Kea). The power law fit to our data set between 10-3 and 10-1 Hz is shown by the line and has an exponential of $-1.78 \pm 0.24$. This represents the average power law for the combined data sets (4-8 minutes in length) taken on this night and the standard deviation of the power law fits to the individual data sequences. The 1-sigma uncertainty in the exponent of each power law fit is better than comparable and has an average value of 0.17 .

To explore the variations at frequencies higher than $\sim 0.1 \mathrm{~Hz}$ one would need to fully account for all of the telescope vibrations. This is a daunting task. Instead, we took advantage of the fact that the laser guide star profile on this night had two strong and distinct peaks. We calculated the relative position of these two peaks within the sodium layer profile (see Figure 1) and then took the differential motion of the two peaks as the parameter of interest. This approach removes telescope and atmospheric jitter though we note that this differential motion is not uniquely linked to the motion of the centroid of the 
layer. For example, the two peaks could move in such a way that the overall centroid does not change at all. However, the timescale for motion of these features does represent large scale changes in the sodium layer so our approach is to compare power laws that describe the differential motion at low and at high frequencies. If they are the same, we have some confidence that the projection of the sodium layer mean motion can be projected from low frequencies to higher frequencies.

To track the position of the two peaks we fitted two 1-dimensional gaussians to each laser guide star profile. The centroid of the two gaussians were allowed to vary to track the position of the peaks but the widths of the gaussians were fixed to the standard deviations found when fitting two gaussians to the mean profile for that data set. As a result we have lost some information on the finest spatial scales since the mean profile has less small scale structure. Typically the standard deviations of the two gaussians fit were 1 and $3.5 \mathrm{~km}$.

As above the individual data packets were combined in to longer data sets. Here the criteria for definition of a data sequence was that there were clearly two peaks and that they remained distinct throughout the data sequence. The power spectra of the data sequences, calculated via a Lomb periodogram ${ }^{4}$ as above, are shown in Figure 4 below. Power laws are fit to the low frequency end $(0.001$ to $1 \mathrm{~Hz})$ of the PSD up to the $1 \mathrm{~Hz}$ where the noise floor becomes dominant.

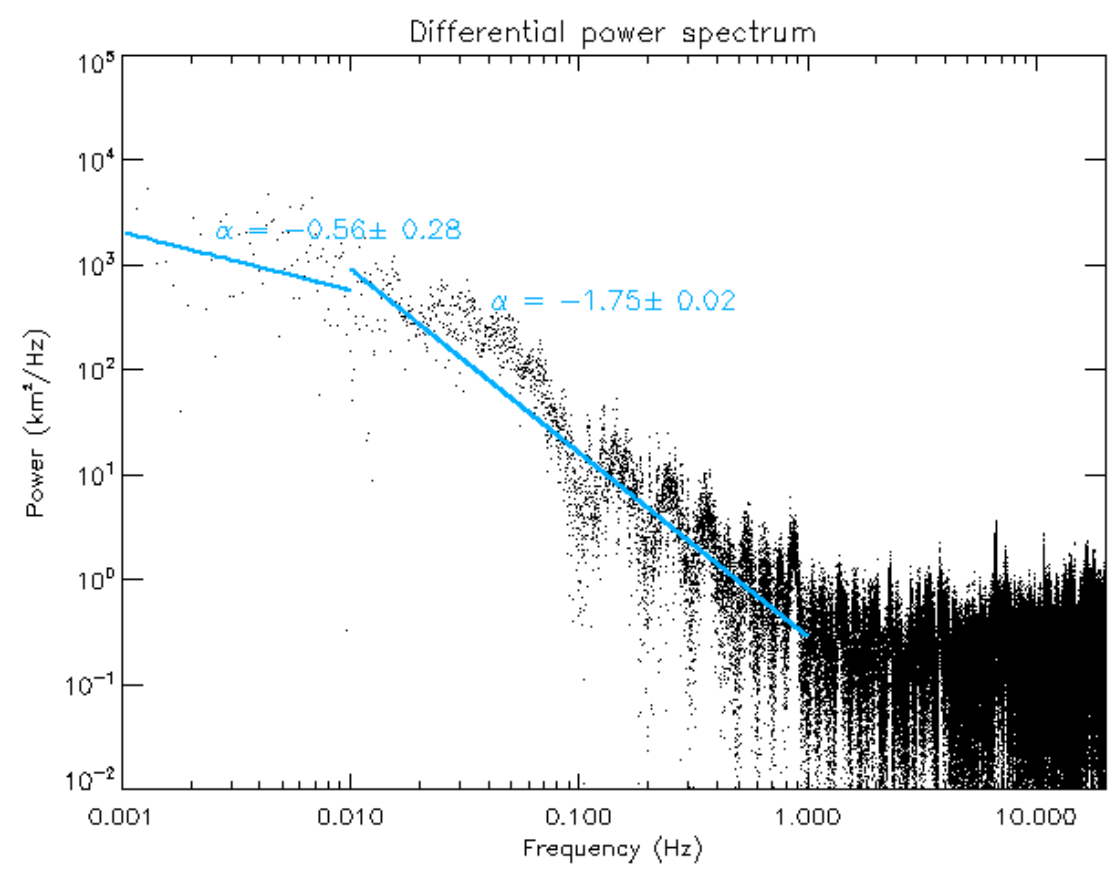

Illustration 4: The power spectrum of the differential motion between two peaks in the sodium layer profile. The power law for intermediate frequencies follows the $\alpha=-1.8$ power law found by the lidar experiments ${ }^{2}$ but exhibits a turn over to a shallower frequencies less than $0.01 \mathrm{~Hz}$. The noise floor of the measurement is visible above $1 \mathrm{~Hz}$. The two fit power laws are fit to the power spectrum from 0.001 to $0.01 \mathrm{~Hz}$ and from $0.01 \mathrm{~Hz}$ to $1 \mathrm{~Hz}$ respectively. The power law exponentials fits and their 1-sigma uncertainties are shown in the figure. Note that this power spectrum consists of all of the data taken that night and the high-frequency range extends to $1 \mathrm{~Hz}$. As a result, there are many more data points in the fit to that power law.

\section{CONCLUSIONS}

Our basic conclusion is that the power spectrum of the mean altitude of the sodium layer altitude continues to follows a simple power law with an exponent of $\alpha=-1.8$ between $0.001 \mathrm{~Hz}$ and $0.1 \mathrm{~Hz}$. 
We find that this shape is in agreement with that found earlier by Davis et $\mathrm{al}^{2}$. Taken together this power law describes the variations over four orders of magnitude (1e-5 to $0.1 \mathrm{~Hz}$ ).

Inherent in the technique used here is telescope shake and drift between the three telescopes (Gemini$\mathrm{N}$, Gemini-N laser launch telescope, and the UH2.2m telescope. This must be removed to detect motion at the sodium layer at higher temporal frequencies (e.g. frequencies where the jitter/drift occurs). Addressing the high-temporal characteristics of the sodium layer mean altitude with this approach is not ideal since the identifying and removing these vibrations is difficult. For this data set we took advantage of the fortuitous fact that there were two distinct features in the sodium layer for which we could calculate their differential positions. Variations in the differential positions of these peaks is a measure of the changes in the sodium layer but are not the quantity of direct interest to adaptive optics systems (e.g. the mean altitude). The fact that the form of the power law for the differential position follows a similar power law from low $(0.01 \mathrm{~Hz})$ to high $(1 \mathrm{~Hz})$ frequencies suggests that the the two exhibit similar temporal characteristics. If true, then the power law of the mean altitude of the sodium layer likely extends along its simple power law to frequencies $1 \mathrm{~Hz}$ as well.

\section{REFERENCES}

[1] G. Herriot, P. Hickson, B. Ellerbroek, and C.Y. She, SPIE 6272.

[2] Davis, D. Saul, Hickson, P., Herriot, G., and She, C.-Y., Optics Letters 2006, Vol 31 No. 22

[3] Butterley, T. et al, 2008, SPIE

[4] Numerical Recipes in C: The Art of Scientific Computing Chapter 13.8, p 581-584. 